\title{
Clinical findings and management of intracranial Hydatid cyst in north of Iraq
}

Accepted: 12/11/2012

\section{Emad Kh. Hammood *}

\begin{abstract}
Background and objective: Where Hydatid cyst of $E$. granulosus reaches $1 \mathrm{~cm}$ in diameter , its wall will differentiated into a thick outer laminated and thin germinal epithelium from which occasionally, daughter cysts could appear within the Hydatid. Aim: management of the Hydatid cyst is performed surgically by separating the two layers of the cyst keeping the inner layer intact during removal process to prevent the generation of daughter cysts at the same site in the future.
\end{abstract}

Methods: We conducted the study using hematology coulter counter to analyze whole blood samples of rats after exposure to welding fumes. We investigated the effect of wielding fume exposure on some hematological parameters such as the red blood corpuscles (RBCs), white blood cells (WBCs) and platelet parameters. Fourteen male albino rats were randomly assigned to control or wielding fume inhalation.

Results: Total removal of the cyst was done in thirty cases using neurosurgical microscope to separate the cyst wall. Two patients had multiple intracranial Hydatid cysts. The commonest location was in the parietal lobe (21 cases). Recurrence was seen in two cases as a result of rapture of the cyst during the first surgery, in other two cases secondary infestation was seen after 1st operation 9-12 months.

Conclusion: Infection acquired in early life and symptoms usually appear later. (mebendazole) is used as prophylaxis. Pathologically there is no calcification of all cysts. radiologically pressure effect is not associated with edema. Big size lead to shift of the brain to the opposite side

Keywords: Hydatid cyst, E. granulosus, mebendazole, headache, brain.

\section{Introduction}

The genus Echinococcus contains three species for which humans are host to the larval stage, or Hydatid. These three species are all found as adult worms in Candidate $^{1}$. A fourth, E. oligarthrus, whose status as a human parasite is disputed, occurs as an adult in our felid.

The eggs ingestion by the intermediate host, the embryos, released from their surrounding membranes by action of the digestive juices, bore actively into the intestinal wall and enters a blood vessel. When humans become accidental intermediate hosts, the larvae penetrate the intestinal wall and spread into the circulation. The larvae may then develop into hydatid cysts in different organs, most commonly in the liver \& lungs ${ }^{1}$. The embryo develops slowly into the Hydatid cyst, reaching a diameter of $1 \mathrm{~cm}$ in 5 months and thereafter enlarging growth depends on location, By the time the cyst of E. granulosus has reached the diameter of $1 \mathrm{~cm}$, its wall is differentiated into a thick outer laminated, no cellular layer, or limiting membrane, which covers the thin germinal epithelium. From the germinal epithelium, masses of cells grow into the cavity of the cyst. Known as the brood capsules. Protoscolices bud from the inner wall of the brood capsule. Occasionally, daughter cysts appear within the hydatid $^{2}$. In North Africa, Iraq, and Iran, hydatid cysts occur with high frequency in the

*Department of General Surgery, College of Medicine, Hawler Medical University, Erbil, Iraq. 
lungs of camels ${ }^{2}$. A number of cases have been reported from California, Arizona, highest in the world Kenya, Ethiopia E. multilocularis is a small tapeworm (1, 2-4, 5 $\mathrm{mm}$ in length) that parasites red and arctic foxes dogs and cats are the definitive hosts. Definitive hosts are always carnivores. In the definitive hosts the adult tapeworm, consisting of 2 to 6 proglottids, lives attached to the luminal surface of the small intestine. The terminal proglottid contains mature eggs (ovoid, 30-40 $\mu \mathrm{m}$ in diameter). The embryonated eggs, the infectious stage, are long-lived and highly resistant to high and low temperature (more than $50^{\circ} \mathrm{C}$ and down to $-40^{\circ} \mathrm{C}$ ). The mature eggs are shed with fasces and are spread in the environment. It is assumed that the intermediate host acquires the infections through the ingestion of contaminated fruits and vegetables ${ }^{1,2}$. Then the intermediate hosts (predominantly rodents or other small mammals, or, accidentally, Humans) ingest eggs, the onchosphere hatches from the egg in the duodenum. The activated oncosphere penetrates the small intestine, enters blood vessels and reaches Primarily the liver via the portal vein; in the liver the oncosphere proliferates into the metacestode surrounded by an inner germinative membrane and an outer laminated layer. The lifecycle is completed when an intermediate host, carrier of viable protoscolices within the cysts, is devoured by a definite host ${ }^{3}$. Our aim in the management of the Hydatid cyst should be surgical \& the way to do that is to separate the two layers of the cyst by neurosurgical microscope \& to keep the inner layer intact during removal to prevent generation of daughter cysts same site in the future.

\section{Methods}

We prospectively analyzed 32 cases of intracranial Hydatid cysts managed between 1998-2009 in Al Rahma private hospital in Mosul, Hawler private hospital and Erbil teaching hospital. We have analyzed their mode of presentation after admission to the hospital. The patients' ages ranged from 6 years to 53 years. Nineteen patients were below 20 years old, six were between 2130 and seven were 31-53 years old, see Table 1. MR imaging findings of intracranial granulosus echinococcosis have been reported to show Well defined oval mass with signal intensities similar to those of CSF, sometimes with a hypo-intense rim4. After the diagnosis of these cases as a hydatid cyst of the brain by C.T scan or M.R.I, they were treated surgically by complete removal of the cyst through craniotomy procedure near the site of the cyst, The commonest location was in Parietal region in about 21 patients, 7 patients in frontal lobe, 3 patients in occipital lobe and 1 patient in the posterior fossa, see Table 2. Intracranial hydatid cysts are commonly seen in the supratentorial compartment located in cerebral hemispheres, other reported sites include subarachnoid space, ventricles pons, cerebellum, aqueduct of Sylvius, extradural space and diploic space of skull bones ${ }^{5}$.

All patients presented with headache, five patients with papillodema, nineteen patients with neurological deficit, twelve patients presented with headache only as an out patients, Table 3 . Clinical features of patients with intracerebral hydatid were not specific. Increased intracranial pressure, epilepsy, neurological disturbances such as dysarthria and hemiparesis, skull deformity and cranial nerve palsies have been reported6. Radiological investigations via CT and MRI have proven to be more helpful diagnostic method. Many asymptomatic Hydatid cysts are first discovered on radiographic examination. The cyst is usually shows a sharp outline by CT \& MRI ${ }^{5,7}$. A number of serologic tests available for the diagnosis of Hydatid disease. The indirect hemagglutination test (IRA) and enzyme-linked immunosorbent assay (ELISA) Serologic cross reactions (used in some patients). The surgical aim (craniotomy) is to separate the outer layer of the cyst from the inner germinal layer through an opening created in the outer layer by using Electrical Microscope (Ziess) and then 
irrigation with saline between two layers, through which hydrostatic pressure lead to the separation of outer from inner germinal layer, which will eventually lead to the delivery of the cyst from the brain slowly through an opening. Complete removal had been achieved except in 2 patients, in which the cyst was ruptured accidentally with no anaphylactic reaction. The space left in the brain by the cyst had been filled with normal saline then closure of the dura. 30 patients had a complete removal of the cyst and only 2 cysts were ruptured (encountered) accidentally during the operation. All 32 patients were followed by C.T scan for 20-36 months after surgery. Recurrence didn't occur except in the two patients through which accidental rupture occured during operation by other neurosurgical center. One patient was a grocery seller who had three surgical interventions previously. The time period between the first and second operation was 8 months and between the second and third operation was 9 months (the site of new cysts were different each time, the 1st. was in the Lt. occipital region \& the second was in the Lt. parietal \& the last was in the Rt. Parietal lobe). Albendazole therapy is given in a daily dose of $10 \mathrm{mg} / \mathrm{kg}$, taken three times for 6 months for all our patients. It is a broad spectrum oral antihelminthic drug. Fourteen patients had associated cysts in the liver, lung, and bone, only one patients had hydatid cysts involving liver, lung, brain, bone \& peritoneum.

\section{Results}

We started with 32 patients in Al-Rahma Hospital \& Hawler private hospital and Erbil teaching hospital in Erbil city. From April 1998 to December 2009, the mean age of patients was 9.4 years ranging from 6 years to 53 years. Following radiological diagnosis, Electrical microscope is used to distinguish the outer layer from the inner layer of the cyst via small hole in the outer layer through which the cyst can be passing out by pressure effect. We noticed that there is no calcification of all cysts .and all cysts were under pressure. Not associated with edema due to the slow growing effect causing brain shift to the opposite side.

We have only two accidental ruptured cysts during separation process. The recurrence after operation was usually noticed by $9-16$ months postoperatively was more commonly associated with midline shift to the opposite side. The second hand surgery showed multi cyst by microscope which exceed 100 cysts at the original site. It is not inert cyst which is proved by cytological examination. Which prove a lot of scoleses inside. Many patients have more than one site of infection in the chest-liver, lung-liver. Only one patient has more one site of infection extra cranially liver, lung, bone even peritoneum. During follow up of these patients by series of MRI or CT scan of the brain, for about 5years every (6-12 months). Three patients (9.3\%) with big size cyst had chronic subdural heamatoma ( SDH) on the opposite site of the operation due to return of the brain to normal position but still followed by gap formation at the same side of manipulation.

There is no other complication as anaphylactic shock, during rupture cyst in the operation only 7 patients $(21.8 \%)$ have epileptic attack more than three times during 6 months after operation and one (3.1\%) patient had CSF leak from the site of the wound Table No.4. -Most of the patients were from rural areas with history of contact animals. Or they were farmers, only one patient had an academic degree employed at a research Lab dealing with $\mathrm{Hy}$ datid. 
Table 1: Patients age.

\begin{tabular}{lcc} 
No. Pt. & Decade (years) & Percentage \% \\
\hline 19 & Below 20 & 59.3 \\
6 & $21-30$ & 18.7 \\
7 & $31-53$ & 21.8
\end{tabular}

Table 2: Intracranial cyst distribution.

\begin{tabular}{lll} 
No. Pt. & Decade (years) & Percentage \% \\
\hline 19 & Below 20 & 59.3 \\
6 & $21-30$ & 18.7 \\
7 & $31-53$ & 21.8
\end{tabular}

Table 3: Signs \& symptoms preoperative

\begin{tabular}{llcc} 
Sign and symptoms & No. Pt. & Percentage \% \\
\hline Papillodema with headache & Hemiplegia & 9 & 15.625 \\
& $\begin{array}{l}\text { Visual } \\
\text { disturbance }\end{array}$ & 7 & 28.12 \\
neurological deficit & behavior & 3 & 21.87 \\
& changes & 3 & 9.37 \\
Headache only & & 12 & 37.5 \\
Epilepsy & & 0 &
\end{tabular}

Table 4: Complications.

\begin{tabular}{lcc} 
Complication & No. Pt. & Percentage \% \\
\hline Rupture intracranial & 2 & 6.2 \\
Infection & 0 & \\
CSF leak & 1 & 3.1 \\
SDH & 3 & 9.3 \\
Recurrence (up to two years) & 2 & 6.2
\end{tabular}




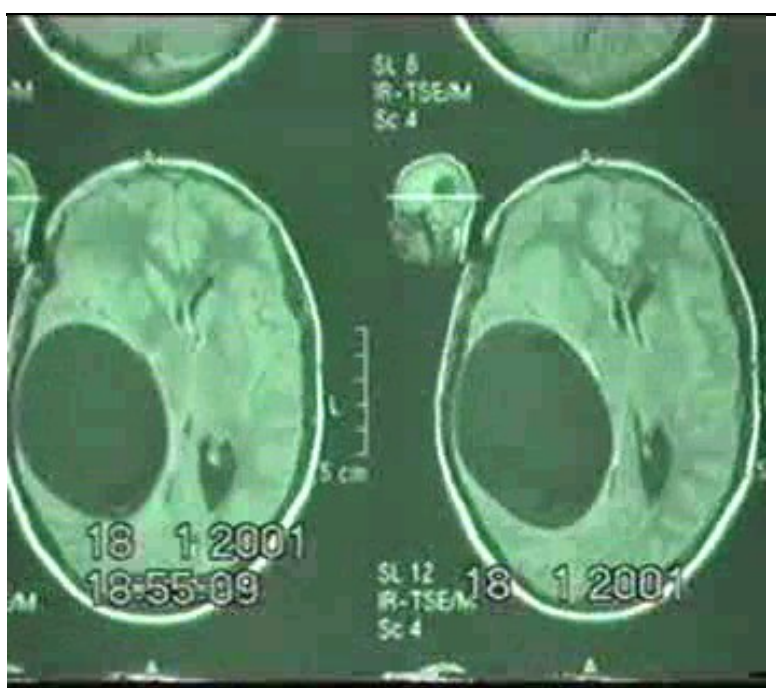

Figure 1: MRI image of the hydatid cyst in the RT. Parital lobe

\section{Discussion}

Intracranial Hydatid disease is rare. The reported incidence is $1-2 \%$ Hydatid disease is endemic in the Middle East, Mediterranean countries, South America, North Africa and Australia8. Cerebral hydatid disease is more common in pediatric population9. Intracranial hydatid cysts are more frequently located in the supratentorial compartment. The parietal lobe is the commonest site and was seen in 21 cases in the present series. The other less common sites reported are skull ${ }^{10}$, cavernous sinus $^{11}$, eyeball ${ }^{12}$, pons ${ }^{13}$, extradural ${ }^{14}$, cerebellum and ventricles ${ }^{15}$. Solitary hydatid cyst in the lateral ventricle is relatively rare site for intracranial hydatid cyst no case report in our study. There is no consensus on the growth rate of the hydatid cyst of the brain and has been variably reported between 1.5-10/year15, New infestation in one of our patient 9 months after 1st surgery during follow up CT scan \& 12 month after 2nd surgery also. Multiple intracranial hydatid cysts, are rare ${ }^{11,15}$ only one case with 2 hydatid at the same site ( frontal lobe)was found in the study. The patients with intracranial hydatid cysts usually present with focal neurological deficit and

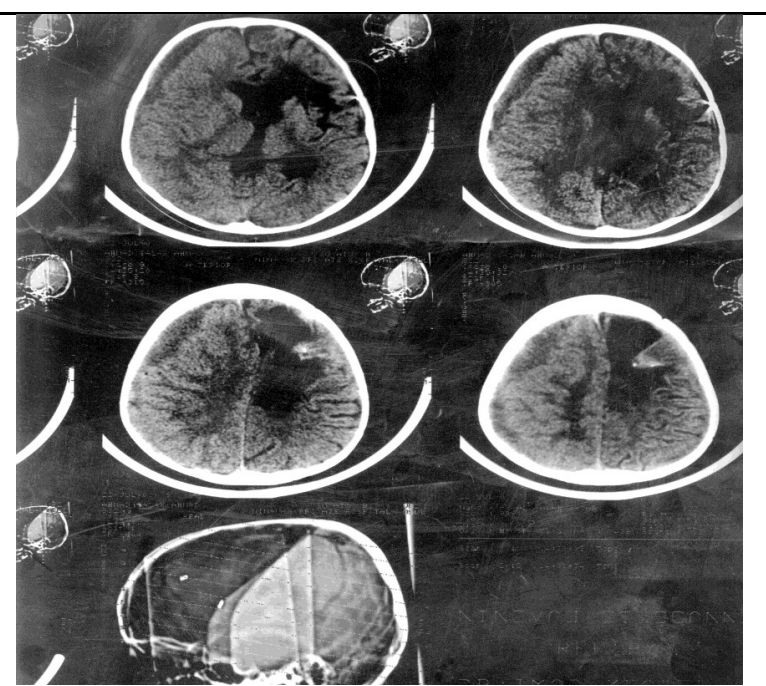

Figure 2: C.T scan image shows RT.

Fronto parital SDH as complication to LT. side surgery

features of raised intracranial pressure due to interference with pathway of CSF flow $^{16}$. All patients in the present series had headache only five patients with papillodema ( increase of intracranial pressure) which is seen in the MRI and CT scan, characteristically show hydatid as a spherical, well defined, non-enhancing cystic lesion without peripheral oedema ${ }^{17}$. Fine rim of peripheral enhancement with perilesional oedema may be seen in the presence of active inflammation ${ }^{18}$. The treatment of hydatid cyst is surgical and the aim of the surgery is to remove the cyst in total without rupture to prevent recurrence and anaphylactic reaction. Rupture is associated with the well recognized problems of anaphylaxis, meningitis, or local recurrence from spillage of the cyst contents ${ }^{19}$. Albendazole therapy is given in a daily dose of $10 \mathrm{mg} / \mathrm{kg}$, taken three times for 6 months. It is a broad spectrum oral antihelminthic drug, which acts by blocking glucose uptake of the larva and adult worm. We found that with the use of Albendazole that is no response to treatment at all \& we had one patient recurrence on different site of previous operation which mean new infestation which not appear in the previous CT preoperative this is against the result of Era 
shin reported better effectiveness of the drug therapy ${ }^{20}$.

\section{Conclusion}

Infection acquired in early life and symptoms appear later. Cyst constitutes 2-3\% of reported cases. The Cyst put as differential Diagnosis of cyst lesion in the brain. Medical management as (mebendazole) used in our study without benefit. The morbidity attributable to the infection depends on the size of the cyst and the site of order. -We noticed that there is no calcification of all cyst .and all the cyst is under pressure. Also it is not associated with edema may be because of slowly growing. Also it leads to shift of the brain to the opposite side when become big size.

\section{References}

1. Tappe, Dennis, August Stich, and Matthias Frosch. "Emergence of Polycystic Neotropical Echinococcosis." Emerging Infectious Disease 2008; 14(2): 292-97. Web. 21 February 2010

2. Eckert, Johannes, and Peter Deplazes. "Biological, Epidemiological, and Clinical Aspects of Echinococcosis, a Zoonosis of Increasing Concern." Clinical Microbiology Reviews 2004; 17 (1): 107-135. Web. 5 February 2010.

3. Canda, M. Serefettin, Merih Guray, Tulay Canda, and Huseyin Astarcioglu. "The Pathology of Echinococcosis and the Current Echinococcosis Problem in Western Turkey." Turk $\mathrm{J}$ Med Sci 2003; 33: 369-374

4. Tunaci M, Tunaci A, Engin G, Ozkorkmaz B, Ahishali B, Rozanes MRI of cerebral alveolar echinococcosis. Neuroradiology 1999;41 844-846

5. Pedrosa I, Saiz A, Arrazola J, Ferreiros J, Pedrosa CS. Hydatid disease: radiologic and pathologic features and complications. Radiographics 2000; 20:795-81

6. Furtado SV, Visvanathan K, Nandita G, Reddy K, Hegde AS: Multiple fourth ventricular hydatidosis. J Clin Neurosci 2009: 16(1):110-112,

7. Algros MP, Majo F, Bresson-Hadni S, Koch S, Godard J, Cattin F, Delbosc B, Kantelip B,. Intracerebral alveolar echinococcosis. Infection, 2003: 31: 63-65.

8. Onal C, Orhan B, Metis O, Three unusual cases of intracranial hydatid cysts in paediatric age group. Pediatr Neurosurg 1997;26:208-13.

9. Jimenez-Mejias ME, Castillo-Ojed E, CuelloContrers JA,. Cerebral hydatidosis. Analysis of a series of 23 cases. Med Clin (Barc) 1991;97:12532.
10. Lunardi $P$, Missori $P$, Di-Lorenzo ND, Cerebral hydatidosis in childhood: a retrospective survey with emphasis on long term follow up. Neurosurgery 1991;29:515-8.

11. Balasubramaniam V, Ramanujam PB, Ramamurthi $B$, Hydatid disease of the nervous system. Neurol India 1970;18:92-5.

12. Rivierez M, El-Azhari A, El-Tantaoui M, Hydatid cyst of cavernous sinus (a case). Neurochirurgie 1992;38:46-9.

13. Sinav S, Demirci, Sinav B, Primary intraocular hydatid cyst. Acta Ophthalmol 1991;69:802-4.

14. Abbassioun K, Amirjmshidi A, Moinipoor MT. Hydatid cyst of the pons. Surg Neurol 1986;26:297-300.

15. Seirra J, Berthier M, Growth rate of secondary hydatid cysts of the brain. J Neurosurg 1985;62:781-2.

16. Nurch G, Francesco F, Montaldo C,. Multiple cerebral hydatid disease: case report with magnetic resonance imaging study. Neurosurgery 1992;30:436-8.

17. Sharma A, Abraham J. Multiple giant hydatid cysts of the brain-case report. Neurosurgery 1982;57:413-5.

18. Karak PK, Mittal M, Bhatia S,. Isolated cerebral hydatid cyst with pathognomonic CT sign. Neuroradiology1992;34:9-10.

19. Ciurea AV, Fountas KN, Coman TC, Machinis TG, Kapsalaki EZ, Fezoulidis NI, Robinson JS: Long-term surgical outcome in patients with intracranial hydatid cyst. Acta Neurochir (Wien) 2006; 148:421-426,

20. Erashin Y, Mutule S, Guzelbag E. Intracranial hydatid cysts in children. Neurosurgery 1993;33:219-24. 